\title{
Conservation communautaire de la forêt humide de basse altitude d'Andriantantely, Madagascar
}

\author{
Maholy Ravaloharimanitra, Hery Nirina Théophile \\ Randriahaingo, Lova Harilala Ranaivosoa, Christelle \\ Chamberlan, Tony King
}

\author{
Correspondence: \\ Maholy Ravaloharimanitra \\ The Aspinall Foundation Programme Madagascar \\ Lot III R 49 Tsimbazaza, 41 Rue Kasanga Fernand \\ Antananarivo 101 - Madagascar \\ E-mail: rrmahooly@gmail.com
}

\section{ABSTRACT}

Andriantantely is a lowland rainforest fragment situated in the Brickaville District of eastern Madagascar, to the south-east of the Ankeniheny-Zahamena forest Corridor. Andriantantely is recognised as a priority site for the conservation of biodiversity in general, and of four species of Critically Endangered lemurs: Prolemur simus, Varecia variegata, Indri indri, and Propithecus diadema. Approximately 4,000 ha in size, the forest extends into five different rural communes, and is impacted by many villages and hamlets, some of which are found within the forest itself. Eight VOI (local community associations) from three communes (Lohariandava, Fanasanagare and Fetraomby) are interested in management transfer contracts for parts of Andriantantely. Since 2010, The Aspinall Foundation works with the Vols for the conservation of Prolemur simus, other lemurs, and their habitats, which helped lead to the removal of $P$. simus from the 25 most endangered primates list in 2012. The conservation strategy was developed following a socio-economic survey undertaken in 2010 which aimed to identify the factors limiting the conservation of the species and its habitat. The main issues identified were poverty of local people and lack of awareness of the value of biodiversity. The first conservation action undertaken was the initiation of a patrol system with local community rangers which increased understanding and protection of the monitored $P$. simus groups. Simultaneously, awareness-raising missions were organised within the communities living close to the $P$. simus sites, aimed at changing local attitudes to natural resources, and particularly lemurs. These initial activities led to the Vols asking the Foundation for assistance in obtaining their management transfer contracts. The first contract was signed in January 2014, but several others need completion, and the development of a long-term conservation strategy for the entire Andriantantely forest, implicating local communities in a true and effective manner, is still required. Environmental education activities have followed the initial awareness-raising missions, to instill practices of rational natural resource use to assure rural development that is respectful of the environment. Much work is still required over a long time period before this rational use might be adopted by the local population in general, by VOI members and non-members alike.

\section{RÉSUMÉ}

La forêt humide de basse altitude d'Andriantantely est située dans le district de Brickaville, au sud-est du corridor AnkenihenyZahamena dans l'est de Madagascar. Andriantantely est reconnu comme site prioritaire pour la conservation de la biodiversité en général, mais également pour celle de quatre espèces de lémurien en Danger Critique d'Extinction, à savoir Prolemur simus, Varecia variegata, Indri indri et Propithecus diadema. Avec une superficie d'environ 4000 ha, la forêt d'Andriantantely s'étend sur cinq communes rurales et concerne plusieurs villages et habitations éparses dont certaines se trouvent en son cœur même. Huit groupements communautaires Vondron'Olona Ifotony (VOI), réparties dans six fokontany appartenant à trois communes rurales du bloc forestier regroupant ces villages, à savoir Lohariandava, Fanasana gare et Fetraomby, sont intéressées par la gestion de la forêt d'Andriantantely. Depuis 2010, la fondation Aspinall (The Aspinall Foundation) a travaillé avec des VoI pour la conservation des lémuriens dont Prolemur simus et de leurs habitats. Son action a permis de retirer $P$. simus de la liste des 25 primates les plus menacés au monde en 2012. Pour ce faire, les axes et stratégies d'intervention ont été élaborés suite à une étude socio-économique menée en 2010. Cette étude a cherché à identifier les facteurs limitant la conservation de l'espèce et la préservation de son habitat. Les principaux facteurs identifiés étaient la pauvreté des Hommes vivant à la périphérie et dans la forêt, ainsi que la méconnaissance de la valeur de la biodiversité. La première étape de l'action de conservation a été la mise en place d'un système de patrouilles assuré par des gardes locaux. Parallèlement aux patrouilles, des campagnes de sensibilisation ont été menées auprès des communautés villageoises pour que leurs attitudes vis-à-vis des ressources naturelles et plus spécifiquement des lémuriens évoluent. Ces travaux de sensibilisation ont porté leurs fruits puisque ce sont les Vol ellesmêmes qui ont sollicité l'appui de la fondation pour l'obtention d'un contrat de transfert de gestion. Le premier contrat a été signé en janvier 2014, mais d'autres sont en cours de réalisation depuis début 2015. L'élaboration d'une stratégie de conservation à long terme pour l'ensemble de la forêt d'Andriantantely, impliquant la population locale de façon réelle et effective, reste à élaborer. Les campagnes d'éducation environnementale ont succédé à celles de la sensibilisation afin d'inculquer les pratiques d'une utilisation rationnelle des ressources naturelles pour 


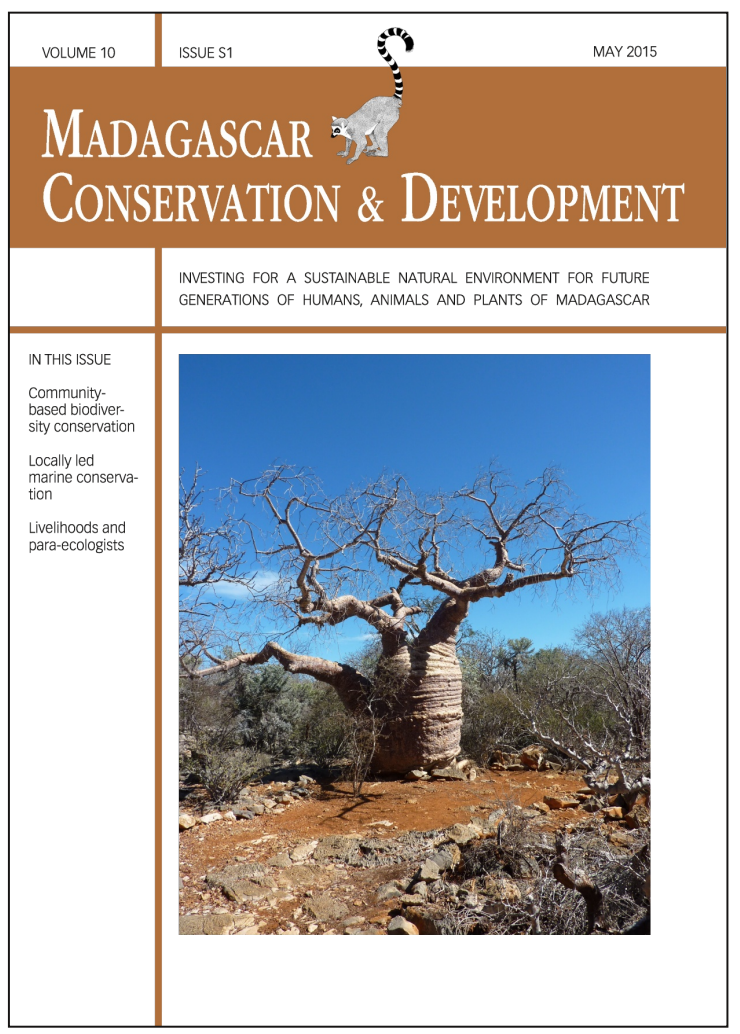

Madagascar Conservation \& Development is the journal of Indian Ocean e-Ink. It is produced under the responsibility of this institution. The views expressed in contributions to MCD are solely those of the authors and not those of the journal editors or the publisher.

All the Issues and articles are freely available at http://www.journalmcd.com

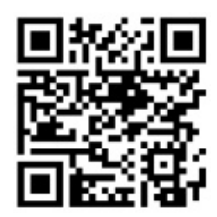

Contact Journal MCD

info@journalmcd.net for general inquiries regarding MCD funding@journalmcd.net to support the journal

Madagascar Conservation \& Development Institute and Museum of Anthropology

University of Zurich

Winterthurerstrasse 190

$\mathrm{CH}-8057$ Zurich

Switzerland

Indian Ocean e-Ink

Promoting African Publishing and Education

www.ioeink.com

Missouri Botanical Garden (MBG)

Madagascar Research and Conservation Program

BP 3391

Antananarivo, 101, Madagascar 
assurer un développement rural respectueux de l'environnement. Un long chemin reste néanmoins à parcourir pour que cette utilisation rationnelle soit pleinement adoptée par l'ensemble de la communauté, que les villages soient membres ou non des organisations communautaires ou VOI.

\section{INTRODUCTION}

La fondation Aspinall (The Aspinall Foundation) est une organisation non-gouvernementale œuvrant à Madagascar depuis 2009 pour la sauvegarde des espèces menacées d'extinction, en particulier les lémuriens (King et Chamberlan 2010). Des missions de reconnaissance des sites pouvant abriter Prolemur simus, l'espèce phare sur laquelle la fondation a initialement travaillé, ont permis de découvrir l'espèce dans une partie du bloc forestier d'Andriantantely, district de Brickaville, à l'est de Madagascar (Ravaloharimanitra et al. 2011). Depuis cette découverte, diverses actions ont été menées, y compris dans les domaines social, économique et environnemental, afin de réduire considérablement les menaces et pressions pesant sur la population de ce lémurien dans ce site et son habitat (Lantovololona et al. 2012).

Les données fossiles et subfossiles ont montré que Prolemur simus avait eu une aire de répartition ancienne bien plus étendue que celle qui était connue récemment (Mahé 1976, VuillaumeRandriamanantena et al. 1985, Godfrey et VuillaumeRandriamananatena 1986, Godfrey et al. 2004). L'espèce a été présumée éteinte suite à l'absence d'observation d'individus au début du 20e siècle (Napier et Napier 1967) mais a été redécouverte dans la partie sud-est de l'île dans les années 1960 et 1970 (Petter et al. 1977, Meier et Rumpler 1987). Une étude collaborative menée en 2009 par la fondation Aspinall, I'Association Mitsinjo, le Groupe d'Étude et de Recherche sur les Primates de Madagascar (GERP) et Conservation International, a permis d'identifier la présence de l'espèce autour de la forêt d'Andriantantely (Ravaloharimanitra et al. 2011).

La fondation s'est alors impliquée dans la gestion de la forêt d'Andriantantely afin de préserver I'habitat des Prolemur simus et assurer leur conservation en même temps que celle des populations de trois autres espèces de lémuriens en Danger Critique d'Extinction (Varecia variegata, Indri indri et Propithecus diadema). Dans un premier temps, ses actions se sont limitées au suivi des groupes de Prolemur simus identifiés, pour dissuader les pratiques de chasse et d'installation de piège ainsi que pour connaître les habitudes des animaux. Ensuite, en réponse à une sollicitation des villageois de la région, elle a appuyé un processus destiné à obtenir un contrat de transfert de gestion aux VOI intéressés par la gestion de la forêt (King et al. 2013b). Finalement, la fondation s'est impliquée dans la mise en œuvre du plan d'aménagement et de gestion afin de veiller au respect du cahier des charges et l'application des mesures répressives connues localement sous le terme de dina pour concrétiser une convention collective portant sur le transfert de gestion. Le présent article décrit les étapes de la collaboration entre la fondation et les organisations villageoises (VOI), usufruitières des ressources naturelles d'Andriantantely.

La VOI (Vondron'Olona Ifotony) est un groupe d'intérêt de personnes appartenant à une organisation communautaire partageant les mêmes intérêts et les mêmes convictions. Dans le cas présent, I'organisation s'engage dans la gestion rationnelle des ressources naturelles, suivant un plan d'aménagement et de gestion établi d'une manière participative et suivant un contrat établi avec l'administration forestière. L'étendue géographique d'un transfert ou d'une proposition de transfert de gestion peut concerner un bloc forestier s'étendant sur une partie ou la totalité d'un ou de plusieurs fokontany (la plus petite division administrative de Madagascar). Les Vol concernées ici, ont ensuite été regroupées en fédération de VOI, à l'initiative de conservation International, généralement à l'échelle des districts. La fédération des VOI du district de Brickaville ou Tsarafaniry est composée des membres des comités de direction de chaque VOI fédérée.

\section{DESCRIPTION DU SITE}

SITUATION GÉOGRAPHIQUE DE LA FORÊT D'ANDRIANTANTELY. La forêt classée d'Andriantantely (E048 46', S18 40') est un bloc de forêt humide de basse altitude, les altitudes étant comprises entre 300 et $700 \mathrm{~m}$. Elle est considérée comme un satellite du corridor forestier d'Ankeniheny-Zahamena, au sudest dudit corridor, à $35 \mathrm{~km}$ au nord-ouest de Brickaville. Le tableau de bord environnemental de la région Atsinanana, datant de 2005 et basé sur d'anciennes données de couverture forestière, mentionne une superficie de 5835 ha. D'autres équipes ont estimé sa superficie à 4170 ha (Andriamasimanana et al. 2001, Schmid et Alonso 2005). La diminution de la superficie pourrait être expliquée par la déforestation, conséquence de défrichements (teviala) et de la pratique de la culture sur brûlis (tavy).

Du point de vue administratif, la plus grande partie du bloc forestier d'Andriantantely est localisée sur la commune rurale de Lohariandava, la section nord-ouest sur celle d'Ambohimanana, la partie nord-est sur celle de Fetraomby et la partie sud-est sur celles de Fanasana gare et Razanaka. Les chefs-lieux des communes rurales de Fanasana gare et de Lohariandava sont desservies par le chemin de fer, anciennement appelé TCE ou Tananarive - Côte Est. Les sites proprement dits ne sont accessibles qu'à pied, le long de sentiers émanant de chaque commune.

LA BIODIVERSITÉ DU BLOC FORESTIER D'ANDRIANTANTELY.

Lors de l'évaluation rapide de la biodiversité organisée par Conservation International en 1998 et 1999 sur cinq sites du Corridor Ankeniheny-Zahamena (CAZ), I'équipe de schmid et Alonso (2005) a souligné le caractère intact et unique de la forêt d'Andriantantely pour la diversité des espèces de la faune et de la flore. L'équipe d'inventaire a recensé une plus grande diversité de lémuriens, de chauves-souris, de micromammifères et rongeurs endémiques que dans les autres sites étudiés. La forêt d'Andriantantely abrite ainsi 64 espèces d'oiseaux, est considérée comme le site le plus important pour la conservation de la diversité des insectes avec 27 familles et six ordres, indicateurs de l'état de la biodiversité. L'herpétofaune est représentée par 72 espèces recensées, dont une espèce d'amphibien et une espèce de reptile appartenant à des taxons non décrits (Schmid et Alonso 2005).

Ces données encourageantes pour mener des actions de conservation ont été renforcées par la découverte récente de Prolemur simus, qui porte à neuf le nombre de lémuriens observés dans cette forêt. Quatre de ces espèces de lémuriens sont classées en Danger Critique d'Extinction par I'UICN : Prolemur simus, Varecia variegata, Indri indri et Propithecus 
diadema (UICN 2015), de sorte que la forêt d'Andriantantely est reconnue comme un des sites prioritaires pour la conservation des lémuriens à Madagascar (King et al. 2013a).

CONTEXTE SOCIO-ÉCONOMIQUE. La forêt s'étendant sur cinq communes et touchant au moins six fokontany et plusieurs villages et habitations éparses, dont certaines se trouvent au cœur même de la forêt (comme c'est le cas d'Ambodihara, commune rurale Lohariandava), le contexte socio-économique est complexe. Sur la base d'une estimation réalisée en 2010 par l'équipe chargée de l'élaboration du Plan de Gestion Environnementale et de Sauvegarde Sociale (PGESS) pour la Nouvelle Aire Protégée Corridor Ankeniheny-Zahamena (NAP CAZ), $18 \%$ de la population totale des trois communes rurales principales concernées par le bloc forestier d'Andriantantely (Ambohimanana, Fetraomby et Lohariandava), pourraient être affectés par le projet de mise en place d'une aire protégée, car dépendants directement ou indirectement des ressources naturelles de la forêt.

Les informations récoltées en 2010 auprès des intervenants dans le domaine de la conservation du district de Brickaville montrent que huit VOI distribuées sur six fokontany appartenant à trois communes rurales, seraient intéressées par la gestion de la forêt d'Andriantantely (Tableau 1). La fondation travaille étroitement avec deux de ces VOI depuis la découverte de Prolemur simus dans le bloc forestier, depuis 2010 et participe notamment à la délimitation des surfaces de transfert de gestion. Le transfert de gestion représente un long travail de collaboration entre l'administration forestière délégataire et le groupe d'intérêt VOI à laquelle la gestion sera transférée.

L'étude socio-économique réalisée dans les villages périphériques de la forêt d'Andriantantely a conclu que l'indice de développement de la population était faible. Lantovololona et ses collaborateurs concluent, en 2012, que ces villages dépendent ainsi étroitement des ressources naturelles. Ces mêmes auteurs ont aussi relevé la présence d'habitations humaines temporaires en lisière de forêt, l'abattage de grandes quantités de bois pour I'usage local, principalement destiné à la construction d'habitations et au bois de chauffe, la collecte de produits forestiers non ligneux, I'installation de pièges, I'utilisation de sarbacanes pour la chasse et enfin le passage en forêt des villageois accompagnés de chiens. La situation politique instable que Madagascar a connue entre 2009 et 2013 n'a pas favorisé la

TABLEAU 1. Récapitulatif des VOI demanderesses ou bénéficiaires de Transferts de Gestion

\begin{tabular}{|c|c|c|c|c|c|}
\hline VOI & $\begin{array}{l}\text { Commune, Fonkontany, } \\
\text { Siège de la VOI }\end{array}$ & $\begin{array}{c}\text { Localisation des } \\
\text { ressources }\end{array}$ & \begin{tabular}{|c|}
$\begin{array}{c}\text { Surface transférée } \\
\text { ou à transférer } \\
\text { (ha) }\end{array}$ \\
\end{tabular} & $\begin{array}{c}\text { Nombre de } \\
\text { membres }\end{array}$ & $\begin{array}{l}\text { Etape du } \\
\text { transfert de } \\
\text { gestion }\end{array}$ \\
\hline Dimbiazanjafy & $\begin{array}{l}\text { Fanasana, Lanonana, } \\
\text { Lanonana }\end{array}$ & \begin{tabular}{|l|} 
Andriantantely/ \\
Marodimatika
\end{tabular} & 310 & 70 & $22 \mid 2014$ \\
\hline Soafaniry & $\begin{array}{l}\text { Fetraomby, } \\
\text { Ambinaninifanasana, } \\
\text { Ambodimanga }\end{array}$ & Andriantantely & * & 75 & En cours 2015 \\
\hline Mevasoa & $\begin{array}{l}\text { Fetraomby, } \\
\text { Ambohimazava, } \\
\text { Ambohimazava }\end{array}$ & \begin{tabular}{|l} 
Andriantantely / \\
Belambo
\end{tabular} & ** & ** & ** \\
\hline Zarasoa & $\begin{array}{l}\text { Fetraomby, } \\
\text { Ambinaninifanasana, } \\
\text { Ampasimiady }\end{array}$ & Andriantantely & 100 & * & $\begin{array}{c}\text { Projet de fusion } \\
\text { avec d'autres } \\
\text { VOI }\end{array}$ \\
\hline Andriatantely & $\begin{array}{l}\text { Fetraomby, Ambodikily, } \\
\text { Andranomihoatra }\end{array}$ & Andriantantely & 2231 & 24 & ** \\
\hline Sahatony & $\begin{array}{l}\text { Fetraomby, } \\
\text { Ambinaninifanasana, } \\
\text { Sahavily }\end{array}$ & \begin{tabular}{|l|} 
Andriantantely \\
Sahatony
\end{tabular} & 100 & ** & $\begin{array}{c}\text { Projet de fusion } \\
\text { avec d'autres } \\
\text { VOI }\end{array}$ \\
\hline $\begin{array}{l}\text { Soafaniry } \\
\text { Sahamanantsy }\end{array}$ & $\begin{array}{l}\text { Lohariandava, } \\
\text { Ambodiantafana, } \\
\text { Ambodiantafana }\end{array}$ & \begin{tabular}{|l|} 
Andriantantely/ \\
Sahasarotra/ \\
Sahamanantsy \\
\end{tabular} & 1158 & 34 & $30 \mathrm{XI} 2014$ \\
\hline Tambatra & $\begin{array}{l}\text { Lohariandava, } \\
\text { Andonabe, Andonabe }\end{array}$ & \begin{tabular}{|l|} 
Forêt \\
Sahasarotra
\end{tabular} & 694 & 62 & $30 \times 12014$ \\
\hline
\end{tabular}

conservation de la biodiversité. La population s'est appauvrie en dépendant de plus en plus des ressources naturelles.

\section{OBJECTIFS}

Lorsque la fondation Aspinall s'est installée à Madagascar, elle cherchait avant tout à sauver Prolemur simus de la menace d'extinction qui pesait sur l'espèce (King et Chamberlan 2010, Rakotonirina et al. 2011), conformément à sa philosophie, en collaborant avec les partenaires de la place et acteurs engagés à différents niveaux dans la protection des lémuriens et de leurs habitats. Les membres des VOI peuvent être considérés comme les principaux acteurs locaux, car ils vivent à proximité de la forêt et qu'ils sont les principaux concernés par la conservation des ressources naturelles et de leur utilisation pérenne. Le présent article décrit ainsi la mise en place d'un système de gestion communautaire des ressources naturelles du bloc forestier d'Andriantantely, pour la conservation des lémuriens et de leurs habitats.

\section{MÉTHODES}

CONSERVATION DES LÉMURIENS. Pour appréhender localement la situation de Prolemur simus et remporter l'adhésion des riverains au projet de conservation proposé, la fondation Aspinall a commencé par identifier les sites abritant, ou susceptibles d'abriter, l'espèce. Des campagnes d'inventaire ont été menées dans plusieurs communes et auprès de plusieurs VOI à la périphérie et dans le corridor forestier d'AnkenihenyZahamena (Ravaloharimanitra et al. 2011). Les membres des VOI y ont contribué, en partageant oralement leurs connaissances des lémuriens présents dans leur forêt, en proposant une localisation sur une carte topographique des lieux abritant des bambous, et enfin, en proposant des personnes issues de la VOI et pouvant servir de guide à l'équipe de chercheurs. Des vérifications sur site ont été organisées sur la base de ces consultations.

Dans un second temps, un système de patrouille, assuré par des gardes locaux, a été mis en place pour relever des données régulières sur Prolemur simus et son habitat (Ravaloharimanitra et al. 2011). Les VOI ont été chargées d'identifier et de désigner les personnes compétentes et motivées pour assurer les patrouilles. Ces candidats ont été formés en collaboration avec I'Association Mitsinjo, puis par les primatologues de la fondation (Lantovololona et al. 2012). Des rapports de patrouille ont été établis mensuellement et annuellement sur la base des données collectées par les gardes depuis 2010. Depuis 2010, trois patrouilles effectuent un suivi hebdomadaire régulier des groupes de lémuriens identifiés dans trois zones différentes de la forêt.

Des campagnes de sensibilisation destinées à toutes les personnes de tout âge dans la zone à proximité des sites abritant Prolemur simus ont été effectuées (Ravaloharimanitra et al. 2013a). Une attention particulière a été accordée aux écoliers, afin de les inciter à participer activement à la conservation de l'espèce et de son habitat. Des cahiers scolaires ont été distribués pour servir de véhicule d'informations et d'éducation (Chamberlan 2012).

ORGANISATION ET MISE EN EEUVRE DES ACTIONS DE CONSERVATION. Dans un premier temps, la fondation a procédé à l'identification des facteurs responsables de la dégradation des ressources naturelles. Les études socioéconomiques menées par Lantovololona et ses collaborateurs en 
2012 ont montré que les gens de la région dépendaient étroitement des ressources naturelles, au point de les menacer. Ces études ont aussi identifié la présence de VOI dans la zone, mais des VOI peu organisées ou inactives. Raik (2007) a noté que le transfert de gestion des ressources naturelles aux communautés locales pouvait avoir un impact positif sur la conservation à conditions que ces communautés comprennent les intérêts qu'elles peuvent tirer de cette gestion. La réalité sur le terrain est généralement réduite à des VOI qui ont un niveau de connaissances limitées et de faibles capacités de gestion pour élaborer une stratégie. Pour éviter un transfert de gestion peu profitable aux $\mathrm{VOI}$ concernées par la forêt d'Andriantantely, plusieurs réunions d'information ont été organisées avant toute chose pour informer les membres des vol et renforcer leurs compétences afin qu'ils puissent s'organiser pour mieux défendre leurs intérêts tout en respectant l'environnement.

Afin de réduire les adhésions motivées par le seul espoir d'obtenir une aide financière personnelle des bailleurs ou des organismes d'appui, nous avons procédé à une période test de 2012 à 2014. Des réunions d'information, d'éducation et de formation des membres ont été organisées au cours de cette période. Les thèmes abordés incluaient la gestion de la vie associative, les droits, devoirs, obligations et responsabilités des membres vis-à-vis de l'association et de l'association vis-à-vis des parties contractantes du transfert de gestion, la gestion des ressources naturelles. Divers supports didactiques et audiovisuels comme des documentaires, des jeux de rôle, des simulations, ont été utilisés.

Face à des conditions particulières comme lors de cataclysmes naturels, des actions sociales ont été entreprises afin d'aider la population et plus particulièrement pour éviter que la vulnérabilité causée par le cataclysme ne se traduise par une pression démesurée sur les ressources naturelles. Par exemple, après le passage du cyclone Giovanna en 2012, des matériaux pour la réhabilitation des écoles ont été octroyés (Chamberlan et al. 2013). L'objectif poursuivi était de montrer aux gens que les travaux de conservation, malgré leurs exigences et leurs obligations, comportaient aussi leur part d'avantages mais que ceux-ci devaient profiter à la collectivité. La fondation a aussi doté les vol de matériels pour mettre en œuvre les formations dispensées et instaurer une méthode de gestion documentée et archivée.

IMPLICATION DES AUTORITÉS LOCALES ET COORDINATION DES ACTIVITÉS AVEC LES INTERVENANTS. L'organisation de la société malgache dans les zones reculées de Madagascar repose encore sur la hiérarchie d'aînesse. Les communautés vivant à la périphérie de la forêt d'Andriantantely ne font pas exception et l'autorité traditionnelle prime sur toute autre forme d'autorité, y compris celles liées aux lois et règlements. II était donc primordial de convaincre les aînés pour transmettre efficacement les messages de sensibilisation à la conservation de la biodiversité. Des relations privilégiées ont donc été tissées avec cette autorité morale en l'associant à chacune des étapes du projet de conservation et en lui demandant de valider chacune des décisions prises.

Le contrat de transfert de gestion est un contrat passé entre I'administration forestière et la VOI. La mairie fait partie de la commission d'enquête préalable à la signature du contrat et joue le rôle d'interface entre l'administration forestière et la VOI. Elle joue également le rôle de conseil et de recours administratif en cas de conflit durant la phase de gestion. Au niveau local et dans le cadre du transfert de gestion des ressources naturelles, la mairie est représentée par le fokontany. Faute de moyens techniques et administratifs, l'administration forestière n'est pas capable d'assumer toute seule les travaux préliminaires à un transfert de gestion. De son côté, la VOI n'est pas en mesure de supporter les frais engendrés par la mobilisation des agents de I'administration pour la régularisation et l'officialisation du contrat de transfert. La fondation a alors établi une collaboration avec l'administration forestière pour mettre en œuvre et accélérer le processus de transfert.

À I'obtention d'un contrat de transfert de gestion des ressources naturelles, les Vol doivent soumettre leur dina pour homologation auprès du tribunal. Le dina est un règlement qui formule les sanctions portant sur chaque type d'infractions au cahier des charges, et constituant ainsi un document clé du contrat de transfert. À I'obtention de cette homologation, les sanctions définies peuvent immédiatement être appliquées par la VOI, sans recours à des Officiers de la Police Judiciaire. Le rôle de la fondation consistait à appuyer l'application du dina. Les procédures administratives auprès du tribunal étant extrêmement longues, il est quasiment impossible d'obtenir I'homologation d'un dina lors d'un premier contrat de transfert. Pour pallier à ce problème, la fondation a établi une collaboration avec la gendarmerie.

Compte tenu de la diversité des intervenants dans le district de Brickaville, des réunions mensuelles ont été organisées à partir de 2011 pour assurer la coordination des interventions de conservation. II s'agissait d'harmoniser les interventions de chaque entité et d'échanger des informations pour éviter toute confusion au sein des VOI. Ces réunions ont ensuite été reprise à un rythme trimestriel par la direction régionale en charge de la conservation de l'Environnement et des Forêts de la région Atsinanana. Au niveau local, ce rôle d'interface a été assuré par la fédération des Vol, connue sous le nom de Tsarafaniry pour le district de Brickaville.

\section{RÉSULTATS}

Les patrouilles régulières qui collectaient des données dans chaque site ont permis l'amélioration des connaissances sur Prolemur simus, sans oublier que les passages réguliers en forêt ont aussi fait baisser le piégeage et autres pressions anthropiques sur l'habitat de ce lémurien. Randriahaingo et ses collaborateurs (2014) ont noté que le nombre d'individus de P. simus observés dans les huit groupes, suivis régulièrement autour d'Andriantantely, était passé de 48 en 2011 à 103 en 2014. Ces patrouilles ont également permis la création d'emplois pour quelques personnes vivant à la périphérie des sites en améliorant ainsi leur situation économique.

Les activités de sensibilisation déployées depuis 2010 ont permis aux VOI de comprendre l'intérêt d'obtenir des contrats de transfert de gestion, ce qu'elles ont formulé en 2012. La procédure a été entamée à partir de cette période. Les différentes étapes à suivre lors du processus du transfert de gestion, décrites par King et ses collaborateurs (2013b), sont primordiales car elles sont les conditions de son effectivité. II s'agissait de mettre en œuvre les appuis nécessaires pour augmenter les compétences afin que la VOI puisse acquérir son autonomie pour prendre des décisions rationnelles et efficientes, même si les termes du 
contrat et du plan d'aménagement et de gestion reflètent souvent les aspirations de l'organisme d'appui.

Les VOI font souvent remarquer qu'un contrat signé avec des exploitants forestiers est plus avantageux qu'un contrat conclu avec des organismes de conservation de la nature. En effet, pour pouvoir évacuer ses produits, un exploitant forestier aménage ou réhabilite des accès au village. Ces accès permettent aussi à la population locale d'évacuer ses propres produits agricoles. En outre, les travaux d'abattage, transformation et évacuation des produits forestiers, constituent une source temporaire de revenus pour la population locale. Pour assurer la conservation de la nature, il est ainsi primordial de convaincre la population locale de se projeter sur le long terme et leur montrer l'importance du capital naturel par rapport à un avantage immédiat mais éphémère. Au cours des actions menées, certains décideurs administratifs ont opté pour le profit immédiat. De plus, malgré la collaboration avec l'administration forestière, mais à cause de I'étendue de ses responsabilités, seules trois VOI prétendant à la gestion du bloc forestier, avaient obtenu un contrat de transfert de gestion en 2014 et avaient été sensibilisée aux textes et législations relatifs au transfert de gestion, à la gestion des ressources naturelles et à la biodiversité.

La période de 2012 à 2014 a constitué la période test pour la VOI Dimbiazan-jafy, celle dont I'obtention du contrat a été appuyée par la fondation. Cette VOI admettait que jouir de l'usage de la forêt et des produits forestiers constituaient un profit d'une très grande valeur, et qu'en contrepartie de cette jouissance, elle avait le devoir de gérer la forêt de manière pérenne pour satisfaire les besoins du présent et du futur. Cependant le nombre de membres de cette VOI est allé en diminuant, de 70 personnes en 2012 à 30 personnes 2014. Ne sont restées que les personnes réellement motivées par la gestion durable des ressources naturelles de leur localité.

Les réunions trimestrielles de coordination des interventions, dans le domaine de la conservation de l'environnement et des forêts, ont permis d'identifier les huit Vol prétendant à la gestion du bloc forestier d'Andriantantely (Tableau 1). Elles ont également permis aux intervenants d'une même zone de se connaître et de communiquer, mais elles ont été momentanément suspendues à partir de 2012, suite aux problèmes politiques au niveau national. La fédération Tsarafaniry, mise en place par conservation International, est supposée prendre le relais et jouer le rôle d'interface entre les VOI et les bailleurs. Cette fédération s'est trouvée fragilisée car la plupart des VOI qui la constitue, en particulier celles en dehors du bloc forestier d'Andriantantely, sont encore très fragiles et peu organisées. De plus, le texte régissant ces fédérations est encore flou, fragilisant encore plus la structure.

\section{CONCLUSION}

La forêt d'Andriantantely est non seulement remarquable en termes de diversité biologique, mais également en termes d'endémicité (Schmid et Alonso 2005). Les personnes vivant à la périphérie de cette forêt ignorent cependant son importance biologique et pratiquent des activités illicites ayant des impacts négatifs sur les ressources naturelles de cette forêt qui s'en trouve ainsi menacée.

Des groupes d'intérêt que sont les VOI avec les usagers de la forêt ont été constitués afin de réguler l'accès aux ressources naturelles mais, faute de moyens, la plupart de ces groupes n'avaient toujours pas officialisés en 2014 et ne profitaient pas d'un contrat de transfert de gestion. Le processus ne devrait cependant être accéléré inutilement. II faut en effet que les vol deviennent les acteurs réels de la conservation des ressources naturelles en intégrant le développement local. Les communautés villageoises doivent comprendre leur intérêt dans un système générateur de développement et de profits. Elles doivent pouvoir distinguer le transfert de gestion du transfert de biens et assimiler que la possibilité d'un profit personnel ne doit pas être la principale motivation pour adhérer à la VOI .

La mise en place d'une patrouille de surveillance a permis d'améliorer les connaissances et le niveau de conservation des groupes de Prolemur simus sur divers sites de la forêt d'Andriantantely et sur sa périphérie. Dans un avenir immédiat, l'extension de ces patrouilles de suivi à d'autres espèces et d'autres territoires du bloc forestier est recommandée (King et al. 2013a), sans oublier de considérer sérieusement une gestion à long terme, impliquant la population locale de manière réelle et effective.

Le paiement des services environnementaux est une option proposée pour assurer la conservation de la biodiversité. Les personnes des communautés locales doivent préalablement prendre conscience de la valeur de la biodiversité qui les entoure et des services qu'elle leur fournit, consentent à la respecter et à la préserver pour leur plus grand bien. Les campagnes de sensibilisation doivent participer à cet aspect. L'éducation environnementale s'appuyant sur les connaissances acquises localement est un levier efficace pour travailler sur cet aspect. Enfin, ces personnes doivent valoriser au mieux les aides reçues et les avantages des mesures adoptées afin d'en profiter pleinement et d'en comprendre l'impact sur leur vie quotidienne.

\section{REMERCIEMENTS}

Nous adressons nos vifs remerciements à toutes les personnes et institutions publiques ou privées ayant contribué, de près ou de loin, à la réalisation de ce projet, en particulier le Ministère en charge de l'Environnement et des Forêts qui nous a délivré le permis de recherche et a consenti aux transferts de gestion du site aux diverses Vol par le biais de la Direction Régionale en charge de l'Environnement et des Forêts de la Région Atsinanana. Nous tenons également à remercier vivement les représentants des hiérarchies administratives et traditionnelles locales, en particulier Monsieur le Chef de District de Brickaville et Monsieur le Maire de la Commune Rurale de Fanasana Gare, ainsi que les Présidents des fokontany. Enfin, notre reconnaissance sincère va à tous les patrouilleurs locaux pour leur collaboration et compréhension. Les activités ont été financées en principal par The Aspinall Foundation et nous remercions d'ores et déjà The Mohamed bin Zayed Species Conservation Fund (projet 14258776) et la fondation Beauval Nature pour le début de leur soutien de nos projets de conservation dans la forêt d'Andriantantely. Et enfin, et non la moindre, nous remercions les rapporteurs anonymes qui ont consacré une grande partie de leur temps et de leur savoir pour évaluer cette contribution, et dont les critiques ont permis l'amélioration de la version antérieure du présent article 


\section{RÉFÉRENCES}

Andriamasimanana, R. H., Rabenandrasana, M. N., The Seing Sam, V. R., Virginie, M. C., Ratelolahy, J. F. et Rakotonirainy, E. O. 2001. Effets de la fragmentation de la forêt humide sur les populations d'oiseaux et de lémuriens dans le Corridor Mantadia-Zahamena. Lemur News 6: 18-22.

Chamberlan, C. 2012. Sauver Prolemur simus - 3000 cahiers scolaires "Godroka" distribués autour du CAZ. Lemur News 16: 26

Chamberlan, C., Ranaivosoa, L., Ravaloharimanitra, M., Randrianarimanana, H. L., Randriahaingo, H. N. T., Roullet, D. \& King, T. 2013. Distribution of school reconstruction materials following Cyclone Giovanna to local communities working to conserve greater bamboo lemurs in and around the Ankeniheny-Zahamena Corridor, eastern Madagascar. Lemur News 17: 2-4.

Hockley, N. J. \& Andriamarovololona, M. M. 2007. The economics of community forest management in Madagascar: is there a free lunch? USAID, Antananarivo, Madagascar. <http://ow.ly/MS6cm> accessed 10/04/2014.

King, T. \& Chamberlan, C. 2010. Conserving the Critically Endangered greater bamboo lemur. Oryx 44, 2: 167. (doi:10.1017/S0030605310000207)

King, T., Rasolofoharivelo, T., Randrianasolo, H., Dolch, R., Randrianarimanana, L. \& Ratolojanahary, T. 2013a. Andriantantely. In: Lemurs of Madagascar: A Strategy for their Conservation 2013-2016, pp 69-70. IUCN/SSC Primate Specialist Group, Bristol Conservation and Science Foundation, and Conservation International, Bristol, UK.

King, T., Ravaloharimanitra, M., Randrianarimanana, H. L. L., Rasolofoharivelo, M. T. \& Chamberlan, C. 2013b. Community-based conservation of critically endangered lemurs at the Sakalava and Ranomainty sites within the Ankeniheny-Zahamena rainforest corridor, eastern Madagascar. Lemur News 17: 63-70

Lantovololona, F., Bonaventure, A., Ratolojanahary, T., Rafalimandimby, J., Ravaloharimanitra, M., Ranaivosoa, P., Ratsimbazafy, J., Dolch, R. et King, T. 2012. Conservation de Prolemur simus autour de la forêt de basse altitude d'Andriantantely, District de Brickaville. Lemur News 16: 7-11.

Raik, D. 2007. Forest management in Madagascar: an historical overview. Madagascar Conservation \& Development 2, 1: 5-10 (doi:10.4314/mcd.v2i1.44123)

Rakotonirina, L., Rajaonson, A., Ratolojanahary, T., Rafalimandimby, J., Fanomezantsoa, P. et al. 2011. New distributional records and conservation implications for the critically endangered greater bamboo lemur Prolemur simus. Folia Primatologica 82, 2: 118-129. (doi:10.1159/000330910)

Randriahaingo, H. N. T., Ravaloharimanitra, M., Randrianarimanana, H. L., Chamberlan, C., Ratsimbazafy, J. et King, T. 2014. Étude et conservation de Prolemur simus aux alentours de la forêt de basse altitude d'Andriantantely, Madagascar. Lemur News 18: 67-72.

Ravaloharimanitra, M. \& King, T. 2012. First community-managed reserve for greater bamboo lemurs inaugurated. Lemur News 16: 26.

Ravaloharimanitra, M., Ratolojanahary, T., Rafalimandimby, J., Rajaonson, A., Rakotonirina, L.et al. 2011. Gathering local knowledge in Madagascar results in a major increase in the known range and number of sites for critically endangered greater bamboo lemurs (Prolemur simus). International Journal of Primatology 32: 776-792. (doi:10.1007/s10764-011-9500-4)

Ravaloharimanitra, M., Ranaivosoa, L., Chamberlan, C. et King, T. 2013a. Sensibilisation à la conservation de Prolemur simus dans le District de Brickaville. Lemur News 17: 6-8.

Ravaloharimanitra, M., Ranaivosoa, L., Mihaminekena, T.H., Chamberlan, C. et King, T. 2013b. Conservation communautaire de Prolemur simus à Ambalafary, District de Brickaville, Madagascar. Lemur News 17: 54-57.

Schmid, J. et Alonso, L. E. (eds.) 2005. Une évaluation biologique rapide du corridor Mantadia-Zahamena à Madagascar. Bulletin RAP d'Évaluation Rapide 32. Conservation International. Washington, DC.

\section{SUPPLEMENTARY MATERIAL.}

AVAILABLE ONLINE ONLY.

Figure S1. Localisation de la forêt d'Andriantantely

Figure S2. Localisation des fokontany et villages proches de la

forêt d'Andriantantely 